\title{
A study of sexual dysfunction and its associated factors among women in childbearing age, Egypt
}

\author{
Mirfat Mohamed Labib El-Kashif*1 ${ }^{1}$ Sheren Ebrahim El-tahry ${ }^{2}$ \\ ${ }^{1}$ Maternity, Obstetric and Gynecological Nursing Department, Faculty of Nursing, Port Said University, Port Said, Egypt \\ ${ }^{2}$ Medical-Surgical Nursing Department, Faculty of Nursing-Port Said University-Port Said, Egypt
}

Received: September 29, 2018

Accepted: November 20, 2018

Online Published: November 26, 2018

DOI: $10.5430 /$ jnep.v9n3p95

URL: https://doi.org/10.5430/jnep.v9n3p95

\begin{abstract}
Background and objective: Sexual dysfunction is a significant public health problem that is more common among women than in men. The women sexual dysfunction and the factors influencing it have not yet been fully investigated. Aim: The present study aimed to evaluate sexual dysfunction and its associated factors among women in childbearing age, Egypt.

Methods: A population-based cross-sectional survey involving 780 non-pregnant sexual active females aged 15-51 years old who were recruited into the study by random sampling. The study population was selected from health facilities which affiliated to the ministry of health in Damietta Governorate. Interview tools used to collect data related to female and male partner.

Results: A total of 780 women in childbearing age were enrolled. More than half of the studied women have sexual dysfunction versus $46.9 \%$ without sexual function. $22.3 \%$ of sexual dysfunction women have mild dysfunction, $18.5 \%$ of them have moderate dysfunction, and $12.3 \%$ have severe dysfunction. Desire disorder was the most common sexual disorder among participants $(67.3 \%)$, followed by arousal disorder $(55.8 \%)$. There was a statistically significant correlation between female sexual dysfunction and husband's age, educational level and husbands suffer from depression, heavy smokers and hypnotic drugs used. Female sexual dysfunction was found among older age, low educated and non-workers females, as well as women, suffer chronic disease, genital inflammation, and stress.

Conclusions: Sexual dysfunction is prevalent among women, so further study based on a comprehensive survey is necessarily needed.
\end{abstract}

Key Words: Female, Reproductive age, Sexual dysfunction

\section{INTRODUCTION}

Sexual relation is one of the significant factors affecting women's physical and mental health and considered the main aspect of life satisfaction between couples. ${ }^{[1]}$ Sexual health is the integration and coordination of different components for sexual act including physical, mental, emotional and social well-being for all sexual issues and beliefs and not entirely the absence of dysfunction or disease. Female sexual functions include six domains are desire, arousal, lubrication (wetness), orgasm, satisfaction and pain. ${ }^{[2,3]}$ The absence of knowledge and experience regarding sex and insufficient couple communication can cause traumatic reproductive tract problems. ${ }^{[4]}$ Sexual issues are seldom discussed, explored or resolved. Attitudes toward women's sexuality, the role of sexual activity and reproductive health are very strongly influenced by cultural value and restrictions around discussion of sexuality. ${ }^{[5,6]}$

Women's sexual dysfunction (WSD) is a group of disor-

\footnotetext{
*Correspondence: Mirfat Mohamed Labib El-Kashif; Email: drmirfat_k@yahoo.com; Address: Obstetric and Gynecological Nursing Department, Faculty of Nursing, Port Said University, Port Said, Egypt.
} 
ders associated with six domains, and more common among women than men. ${ }^{[2,3,7]}$ The prevalence of women sexual disorders varies among different communities, especially Eastern one, but it is underestimated due to misconceptions of female sexual function and cultural taboos. The prevalence of female sexual dysfunction (FSD) ranges from $26.6 \%$ to $63 \%$ worldwide. It is estimated between $25 \%-76 \%$ in the USA, 76.9\% in Upper Egypt and 46\% in Lower Egypt. ${ }^{[8-10]}$ In another world region, the prevalence of FSD reported $31 \%$ of participants, of which $33 \%$ of them had sexual desire disorder, $16.5 \%$ had arousal disorder, and $25 \%$ had orgasm disorders, while $45.5 \%$ reported painful intercourse. ${ }^{[11]}$ Studies done in China and chorea reported that $50 \%$ of each population experienced sexual dysfunction. ${ }^{[12,13]}$ In Malaysia, $25.8 \%$ of the studied women reported sexual dysfunction with $39.3 \%$ reported hypoactive desire disorder, (25.8\%) arousal problem, (21.5\%) lubrication difficulties, and $(21.5 \%)$ sexual dissatisfaction. ${ }^{[14]}$

In the Arab world, female sexual dysfunction has been inadequately investigated. The findings of a multi-country study done to investigate sexual function in 2920 Arab-speaking women showed that the average prevalence of sexual dysfunction was 59.1\%. ${ }^{[15]}$ In the research done in Saudi Arabia based on the English version of the Female Sexual Function Index (FSFI), the authors reported FSD is more prevalent among young and employed participants. Additionally, Saudi women revealed significantly lower FSFI scores as well as lower scores in four sexual domains. ${ }^{[16]}$

The cause of sexual dysfunction is a multifactorial problem involving biological, psychological, and interpersonal conditions which creates a partial or total loss of the sexual response. Hormonal and physiological factors such as pregnancy, breastfeeding, and menopause play an essential role in reducing sexual excitement. ${ }^{[17,18]}$ Partner's age, duration of the marriage, quality of the relationship, the partner's health status, psychological problems, and how to deal with emotions are considered a fundamental issue for sexual function. ${ }^{[8,19,20]}$

The treatments for sexual disorders are time-consuming, and success in the diagnosis and therapy of female sexual dysfunction has been limited. ${ }^{[21,22]}$ The health care providers should be able to recognize any ambivalent feelings on the part of the patient regarding, sexuality coitus, the partner, body image and the need to have children. Also, they are must be able to disclose serious traumatic experiences and problems. The integration of medical and psychological intervention therapy is significantly improving the manner of a person who received the treatment for sexual dysfunctions. This interventions joins the power of both treatments to aug- ment efficiency, surge treatment, and relational gratification, and reduce patient withdrawal. ${ }^{[23-25]}$

\subsection{Significant of the study}

Women are considered to be more "erotically plastic" than men and hereafter to be more pliable to gender and sexual, cultural proscriptions and beliefs so, women were likely to have a higher incidence of sexual disorders as adults than do men. The sexual dysfunction has a significant impact on women, their relationships as well as their overall quality of life. In daily care practice, nurses faced problems by addressing sexuality, an essential aspect, and necessary in human life that should be appreciated in all health facilities, and all through the lifecycle. ${ }^{[26,27]}$ Nevertheless, the difficulty in addressing, inquiring and replying indeed related sexual problems remains and appears to be a common problem. ${ }^{[28-32]}$ In a study done in Egypt, it was reported that most women $(84.1 \%)$ of sexual problems had not received a professional consultation for their sexual issues. The most reported causes for not seeking to help were: "Embarrassment" (66.7\%) and "Think the doctor cannot help" $(8.9 \%) .{ }^{[19]}$

In Egypt, sexual disorders and unawareness are considered a severe problem. However, this problem is' derived from social pressure and restriction, a shame of and taboo. Therefore, women feel uncomfortable for discussing sexual intercourse and sexuality and could not talk openly about their sexual problems that are why they were not getting help. So, the present study was to evaluate sexual dysfunction and its associated factors among women in childbearing age, Egypt.

\subsection{Aim of the study}

The present study aimed to evaluate sexual dysfunction and determine its related factors among women in childbearing age, Egypt.

\section{SUBJECTS AND METHODS}

\subsection{Study design}

A cross-sectional population-based study design was used.

\subsection{Sampling and data collection}

A sample of 780 married females in the reproductive age were selected after considering the inclusion and exclusion criteria from four general hospitals and eight health care centers PHC which affiliated to the ministry of health in Damietta Governorate, Egypt. A simple random sample selected these Health Care Facilities. The hospitals were old Damietta General Hospital, Kafer Saad General Hospital, Fariskur General Hospital, and Alzarqaa General Hospital. The Health Care Centers PHC were distributed as follows; three health centers in old Damietta, two health centers in 
New Damietta city, one form Kafer Saad city, one form Fariskur city and one from Alzarqaa city. A clustered random sample recruited the study population from gynecology clinics, dermatology, and venereal disease clinics, medical clinics, surgical clinics, and family planning clinics of all selected Health Care Facilities. The sample takes six months to complete from February 2018 to July 2018. The researchers attended one of the selected healthcare facilities three days per week, from 8:00 am. to 12:00 pm.

\subsection{Inclusion criteria}

Sexual active married females in reproductive age, aged 15 to 51 years.

\subsection{Exclusion criteria}

Females with a neurological disorder and psychosis or on antipsychotic drugs, pregnant and breastfeeding women or women at menopause.

\subsection{Tools of data collection}

The interviewing questionnaire: The researcher designed it, consisted of 2 parts:

Part I: Data related to females included: age, parity, mode of delivery, educational level, occupation, body mass index (BMI), practice exercise, type of marriage, frequency of sexual intercourse, menstrual and obstetrical history, circumcision, use of contraception, history of chronic medical conditions and stress. To calculate BMI, women's height and weight were measured, and the value for BMI were stated as follows: a BMI $<18.50 \mathrm{~kg} / \mathrm{m}^{2}$ as under-weight, a BMI of $18.50-24.99 \mathrm{~kg} / \mathrm{m}^{2}$ as normal, a BMI of $\geq 25.00$ $\mathrm{kg} / \mathrm{m}^{2}$ as over-weight and a BMI $\geq 30.00 \mathrm{~kg} / \mathrm{m}^{2}$ was stated as obese. ${ }^{[29]}$

Part II: Data related to male partner were collected from female partners include age, educational level, job. How many wives of the husband, medical condition, personal habits of the husbands, and sexual problems of the husband from the wives point of view depend on medical record and examination.

Female Sexual Function Index (FSFI): It is a standardized questionnaire designed done by Rosen et al. ${ }^{[30]}$ used to evaluate female sexual function. In the present study, it is translated and adapted for the applicability in the community by researchers which it matched with Anis et al. ${ }^{[22]}$ Arabic version. It contains 19 -item questionnaire measures six domains of female sexual functioning during sexual intercourse (desire, arousal, lubrication, orgasm, satisfaction, and pain). The 19 questionnaires were distributed on six domains as the following: desire (items 1,2), arousal (items 3 to 6), lubrication (items 7 to 10), orgasm (items 11 to 13), satis- faction (items 14 to16) and pain (items 17 to 19). Each item score graded from 0 to 5; 0 means no sexual intercourse, 1 indicates sexual dysfunction to 5 indicates normal sexual function. Each woman domain scores are obtained by adding the scores of each woman questions that encompass the domain and multiplying the summation by the domain factor which included in the FSFI for each domain. Factors for desire were $0.6,0.3$ for arousal and lubrication, while 0.4 for orgasm, satisfaction, and pain. The overall score was acquired by adding the six domain scores. The total-scale score range is from 2.0 to 36.0 , and the higher scores suggested better sexual function among women. The sexual domain was considered dysfunction if it is maximum attainable scores were 65 . Hence, scores 3.9 in all six domains were measured as sexual dysfunction. The female sexual function was categorized as four groups: a total FSFI scores Score $\geq 23$ were considered normal female sexual function, a total score from 18 to 23 were mild FSD, total score from 11 to 17 were moderate FSD while total score $\leq$ ten were considered severe FSD. The woman is asked to choose the suitable option for her condition from FSFI during the previous four weeks. All women were examined local and general by specialists as needed. The researchers explain all questions to the women and educate them face to face regarding the cycle of female sexual function and each sexual domain. The questionnaires were completed by the women. For the Illiterate women, the researchers ask questions and then complete the question form.

\subsection{Ethical consideration}

The study established ethical approval from the Research Ethics Approval Committee, Faculty of Nursing, Port Said University. Verbal consent obtained from the participant after full explanation of the aim of the study and ensure confidentially and they have the right to refuse participation.

\subsection{Validity and reliability}

Validity and reliability for the study questionnaire were determined before data collection. Cronbach's alpha test tested the reliability of the questionnaire to measures reliability, or internal consistency. ${ }^{[32]}$ An expert panel of a gynecologist examined the validity of the questionnaire content, dermatology, and venereal disease specialist, medical specialist, obstetrics and gynecology nursing specialist (Ph.D.).

\subsection{Pilot study}

The final questionnaire was firstly tested in a pilot study on 30 women to measure its reliability and applicability, and it has been revealed to be reliable with a Cronbach's alpha as 0.86 . 


\subsection{Handling and analysis of data}

The original data were coded and entered into SPSS system files (SPSS package version 19, Chicago, USA). Analysis and interpretation of data were conducted. The following statistical measures were used; descriptive statistics including frequency, distribution, mean and standard deviation were used to describe different characteristics. Kolmogorov-Smirnov test was used to examine the normality of data distribution. Univariate analyses including $t$-test were used to test the significance of the results of quantitative variables. Chi-Square test, Monte Carlo test and Fisher's Exact test were used to testing the significance of the results of qualitative variables. Odds ratio and $95 \%$ confidence in- terval was calculated for different parameters of the sexual dysfunction scale. The significance of the results was at the $5 \%$ level of significance.

\section{RESULTS}

More than half of the studied women have sexual dysfunction (SD) versus $46.9 \%$ without SD. $22.3 \%$ of sexual dysfunction women have mild dysfunction, $18.5 \%$ of them have moderate dysfunction, and $12.3 \%$ of them have severe dysfunction (see Figure 1). Desire disorder was the most common sexual disorder among participants (67.3\%), followed by arousal disorder $(55.8 \%)$ (see Figure 2).

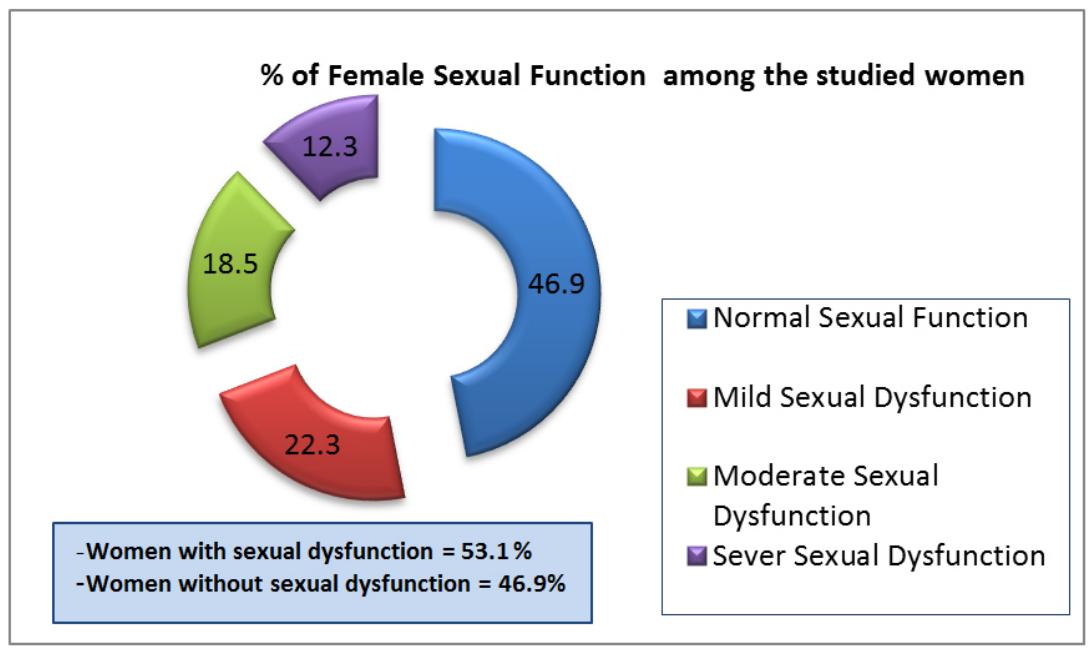

Figure 1. Distribution of the studied sample according to their sexual function $(n=780)$

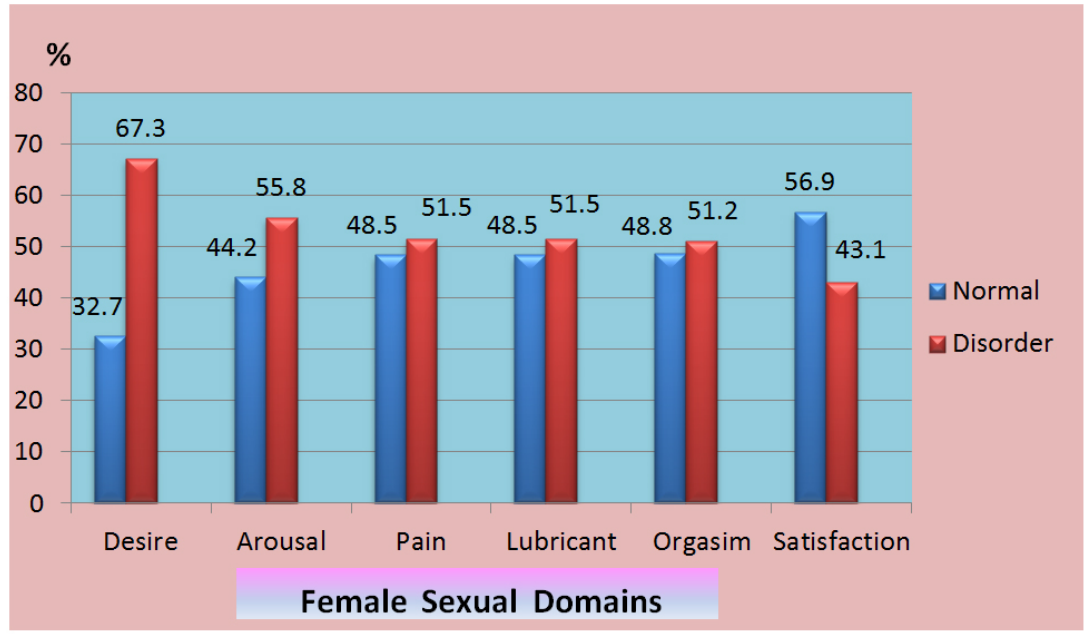

Figure 2. Distribution of the studied sample according to their sexual domains $(n=780)$

The mean age of women with SD (34.8) was significantly higher than of those without SD (32.5). The educational level and job were significantly correlated with the extent of
(SD); nearly one-third of women without SD has postgraduate education, compared only $13.8 \%$ of women with SD. Also, the majority of women without SD (74.6\%) has a job, 
compared to $59.4 \%$ of those with SD. There is no statistically a difference regarding BMI between the two groups. Also, the mean age of husband of a woman who has SD (41.3) was significantly higher than of another group (37.8). Illiteracy was higher among husband of women who has SD, while university and postgraduate education were higher among husband of the woman who has not to have SD. Type of husband job was not statistically significantly associated with FSD. On the other hand, the traditional type of marriage was significantly high among women with SD. Have sex once a month was more frequent among women with $\mathrm{SD}$, while daily sex was more frequent among women without $\mathrm{SD}, p=$ .001 (see Table 1).

Table 2 shows that the differences in the menstrual and obstetric parameters among the two groups were statistically insignificant. Most of the women of two groups have regular menses. The majority of women of the two groups were multipara, and most of them had no abortion. Also, less than two-thirds of women in the two groups have no circumcision and about one-fourth of circumcised women in two groups have a simple degree of circumcision. Vaginal delivery $(50.7 \%)$ was significantly higher among women with SD, compared to $(30.3 \%)$ of another group, as was episiotomy. Type of contraceptive used and female circumcision was not statistically significantly associated with SD.

Table 3 represents that, medical condition as diabetes mellitus, hypertension, and thyroid disorder were significantly high among women with SD. Also, medication use for chronic disease, Inflammation of the genital tract and stress were significantly high among women with SD. Furthermore, Depression, cigarette smoking and hypnotism were significantly high among husband of women who has SD. Mainly, low desire, difficult erection, difficult keeping erection, inhibition or delayed ejaculation and retrograde ejaculation were significantly high among husbands of women who have SD.

Table 4 shows the results of the multiple logistic regression analysis achieved from the final model for each sexual domain, and it was done to realize the best interpreter of sexual domain disorder among women. Disorder of women sexual domains was significantly correlated with increasing age of women and low level of education, although high-level education also showed a significant association. Professional work of women was significantly associated with sexual domain disorder. Regarding obstetrics parameters, high parity was a highly significant variable for (desire, orgasm and satisfaction disorders), as was increased number of pregnancies.

Furthermore, vaginal delivery was significantly associated with sexual domain disorder except satisfaction and pain domains were not reaching the level of significance. This table

Published by Sciedu Press also indicates that a woman has an increasing number of children, and has children less than one year has sexual domain disorder, although five and ten years also showed a significant association. Also, a woman who suffers from chronic diseases and takes medication of these diseases has sexual domain disorder as does the woman suffers from genital tract inflammation and stress.

\section{DiscuSSION}

Female sexual dysfunction is a complex condition and difficult to understand that can affect females of all ages and has negative impacts on their quality of life. Female sexual dysfunction has been a cyclic process that accentuates hormonal, psychological, biologic, environmental and social factors. The research on female sexual dysfunction has gained force in this time because sexual dysfunction is an illness of its entity and is prevalent in all populations. ${ }^{[33,34]}$ In the Arab world, culture proscribes openly discussing sexual issues, and this also relates to Egypt. Sexual problems are not declared likely because women felt shy and refused to mention sexual problems. ${ }^{[35]}$ Deficiencies of nurses' education, knowledge, and skills, as well as a lack of time and awareness, were the possible reasons for inadequate sexual counseling. ${ }^{[36]}$ In Egypt, the nurses can feel uncomfortable with discussing this matter, afraid that women might become distressed, nervous, or insulted when talking about sex and maybe culturally inhibited. ${ }^{[37]}$

The present study presents epidemiological data on the problem of female sexual problems and its associated factors in Egypt, represented by a sample taken from Damietta Governorate. In the current study, the prevalence of female sexual dysfunction was $53.1 \%$. $22.3 \%$ reported mild sexual dysfunction, $18.5 \%$ reported moderate sexual dysfunction and $12.3 \%$ of them have severe sexual dysfunction. The prevalence of FSD varied between countries, although FSD is documented as a widespread health problem. The variation may be due to possible environmental and socioeconomic factors, medical and psychological health condition, as well as cultural and racial variances. A study in El-Fayoum city, Egypt the authors reported that sexual dysfunction occurs in $61.2 \%$ of women. ${ }^{[33]}$ In another study done in Egypt, $52.8 \%$ of females reported sexual dysfunction. ${ }^{[35]}$ These variations in results between studies done in Egypt may be due to differences in the characteristic of samples studied and methodology used in data collection by researchers. In a study done in Iran, $27.3 \%$ of females reported sexual dysfunction, containing $6.3 \%$ mild, $20.3 \%$ moderate, and $0.8 \%$ severe sexual dysfunction. ${ }^{[38]}$ In Turkey, the prevalence of female sexual dysfunction was $70.9 \%$ of the participants based on (FSFI). ${ }^{[2]}$ In Ghana, sexual dysfunction in sexually active women was $70 \% .{ }^{[7]}$ 
Table 1. Socio-demographic profile of the studied sample $(n=780)$

\begin{tabular}{|c|c|c|c|c|c|}
\hline \multirow{3}{*}{ Socio-demographic profile } & \multicolumn{4}{|c|}{ Studied women $(n=780)$} & \multirow{3}{*}{ Significance } \\
\hline & \multicolumn{2}{|c|}{$\begin{array}{l}\text { Women without sexual } \\
\text { dysfunction }(n=366)\end{array}$} & \multicolumn{2}{|c|}{$\begin{array}{l}\text { Women with sexual } \\
\text { dysfunction }(n=414)\end{array}$} & \\
\hline & No. & $\%$ & No. & $\%$ & \\
\hline \multicolumn{5}{|l|}{ Age of wife (years) } & \multirow{3}{*}{$\begin{array}{l}t=2.531 \\
p=.012^{*}\end{array}$} \\
\hline Min-Max & \multicolumn{2}{|c|}{$15.0-51.0$} & \multicolumn{2}{|c|}{$18.0-52.0$} & \\
\hline Mean \pm SD & \multicolumn{2}{|c|}{$32.5 \pm 6.6$} & \multicolumn{2}{|c|}{$34.8 \pm 8.2$} & \\
\hline \multicolumn{5}{|l|}{ Educational level of wife } & \multirow{6}{*}{$\begin{array}{l}\chi^{2}=17.898 \\
p=.001^{*}\end{array}$} \\
\hline Illiterate & 18 & 4.9 & 24 & 5.8 & \\
\hline Read and write & 6 & 1.6 & 36 & 8.7 & \\
\hline Secondary education & 120 & 32.8 & 183 & 44.2 & \\
\hline University education & 105 & 28.7 & 114 & 27.5 & \\
\hline Postgraduate education & 117 & 32.0 & 57 & 13.8 & \\
\hline \multicolumn{5}{|l|}{ The job of wife } & \multirow{3}{*}{$\begin{array}{l}\chi^{2}=14.581 \\
{ }^{\mathrm{MC}} p=.006^{*}\end{array}$} \\
\hline Not working & 93 & 25.4 & 168 & 40.6 & \\
\hline Working & 273 & 74.6 & 246 & 59.4 & \\
\hline \multicolumn{5}{|l|}{ BMI $\left(\mathrm{Kg} / \mathrm{m}^{2}\right)$ of wife } & \multirow{5}{*}{$\begin{array}{l}\chi^{2}=1.565 \\
{ }_{\mathrm{MC}} p=.766\end{array}$} \\
\hline Underweight $(<18.5)$ & 0 & 0.0 & 3 & 0.7 & \\
\hline Normal $(18.5-<25.0)$ & 108 & 29.5 & 108 & 26.1 & \\
\hline Overweight $(25.0-<30.0)$ & 126 & 34.4 & 135 & 32.6 & \\
\hline Obese $(30.0-\leq)$ & 132 & 36.1 & 168 & 40.6 & \\
\hline \multicolumn{5}{|l|}{ Age of the husband (years) } & \multirow{3}{*}{$\begin{array}{l}t=3.1600 \\
p=.002 *\end{array}$} \\
\hline Min-Max & \multirow{2}{*}{\multicolumn{2}{|c|}{$\begin{array}{l}23.0-57.0 \\
37.8 \pm 7.4\end{array}$}} & \multirow{2}{*}{\multicolumn{2}{|c|}{$\begin{array}{l}23.0-62.0 \\
41.3 \pm 10.2\end{array}$}} & \\
\hline Mean $\pm \mathrm{SD}$ & & & & & \\
\hline \multicolumn{5}{|l|}{ Educational level of husband } & \\
\hline Illiterate & 33 & 9.0 & 66 & 15.9 & \\
\hline Read and write & 12 & 3.3 & 39 & 9.4 & $\chi^{2}=13.109$ \\
\hline Secondary education & 156 & 42.6 & 183 & 44.2 & $p=.011^{*}$ \\
\hline University education & 111 & 30.3 & 105 & 25.4 & \\
\hline Postgraduate education & 54 & 14.8 & 21 & 5.1 & \\
\hline Job of husband & & & & & \\
\hline None & 3 & 0.8 & 18 & 4.3 & $\gamma^{2}=6781$ \\
\hline Skilled & 138 & 37.7 & 198 & 47.8 & $\begin{array}{l}\chi=0.181 \\
\mathrm{MC}_{p}=075\end{array}$ \\
\hline Semi-skilled & 129 & 35.2 & 111 & 26.8 & \\
\hline Professional & 96 & 26.2 & 87 & 21.0 & \\
\hline Type of marriage & & & & & $\gamma^{2}=19.550$ \\
\hline Traditional & 225 & 61.5 & 354 & 85.5 & $p<.0001^{*}$ \\
\hline Based on the love story & 141 & 38.5 & 60 & 14.5 & \\
\hline Husband has more than on & & & & & $\chi^{2}=0.030$ \\
\hline No & 321 & 87.7 & 366 & 88.4 & $p=.862$ \\
\hline Yes & 45 & 12.3 & 48 & 11.6 & \\
\hline The frequency of sexual int & & & & & \\
\hline Once per month & 9 & 2.5 & 60 & 14.5 & \\
\hline Twice per month & 18 & 4.9 & 36 & 8.7 & \\
\hline Once per week & 72 & 19.7 & 108 & 26.1 & $\chi^{2}=22.318$ \\
\hline Twice per week & 81 & 22.1 & 81 & 19.6 & $p=.001^{*}$ \\
\hline Three to four times per week & 81 & 22.1 & 78 & 18.8 & \\
\hline Once per day & 75 & 20.5 & 36 & 8.7 & \\
\hline More than once per day & 30 & 8.2 & 15 & 3.6 & \\
\hline
\end{tabular}


Table 2. Menstrual and obstetric parameters of the studied sample $(n=780)$

\begin{tabular}{|c|c|c|c|c|c|}
\hline \multirow{3}{*}{ Menstrual and obstetric history } & \multicolumn{4}{|c|}{ Studied women $(n=780)$} & \multirow{3}{*}{ Significance } \\
\hline & \multicolumn{2}{|c|}{$\begin{array}{l}\text { Women without sexual } \\
\text { dysfunction }(n=366)\end{array}$} & \multicolumn{2}{|c|}{$\begin{array}{l}\text { Women with sexual } \\
\text { dysfunction }(n=414)\end{array}$} & \\
\hline & No. & $\%$ & No. & $\%$ & \\
\hline \multicolumn{5}{|l|}{ Regularity of menses } & \multirow{3}{*}{$\begin{array}{l}\chi^{2}=0.764 \\
p=.382\end{array}$} \\
\hline Regular & 285 & 77.9 & 303 & 73.2 & \\
\hline Irregular & 81 & 22.1 & 111 & 26.8 & \\
\hline \multicolumn{6}{|l|}{ Number of pregnancies } \\
\hline None & 42 & 11.5 & 8 & 5.8 & \multirow{4}{*}{$\begin{array}{l}\chi^{2}=8.780 \\
p=.067\end{array}$} \\
\hline $1-2$ & 159 & 43.4 & 147 & 35.5 & \\
\hline $3-4$ & 120 & 32.8 & 141 & 34.1 & \\
\hline 5or more & 45 & 12.3 & 102 & 24.6 & \\
\hline \multicolumn{6}{|l|}{ Number of deliveries } \\
\hline None & 51 & 13.9 & 18 & 6.5 & \multirow{4}{*}{$\begin{array}{l}\chi^{2}=9.040 \\
p=.060\end{array}$} \\
\hline $1-2$ & 189 & 51.6 & 180 & 43.5 & \\
\hline $3-4$ & 99 & 27.0 & 147 & 35.5 & \\
\hline 5or more & 27 & 7.3 & 60 & 14.4 & \\
\hline \multicolumn{5}{|l|}{ Abortions } & \multirow{3}{*}{$\begin{array}{l}\chi^{2}=1.440 \\
p=.486\end{array}$} \\
\hline No & 264 & 72.1 & 270 & 65.2 & \\
\hline Yes & 21 & 27.9 & 144 & 34.8 & \\
\hline \multicolumn{6}{|l|}{ Vaginal delivery } \\
\hline No & 255 & 69.7 & 204 & 49.3 & \multirow{5}{*}{$\begin{array}{l}\chi^{2}=11.124 \\
p=.001^{*} \\
\chi^{2}=6.166 \\
p=.013^{*}\end{array}$} \\
\hline Yes & 111 & 30.3 & 210 & 50.7 & \\
\hline Episiotomy & & & & & \\
\hline No & 303 & 82.8 & 288 & 69.6 & \\
\hline Yes & 63 & 17.2 & 126 & 30.4 & \\
\hline \multicolumn{5}{|l|}{ Cesarean sections } & \multirow{3}{*}{$\begin{array}{l}\chi^{2}=1.830 \\
p=.607\end{array}$} \\
\hline No & 144 & 39.3 & 186 & 44.9 & \\
\hline Yes & 222 & 60.7 & 228 & 55.1 & \\
\hline \multicolumn{6}{|l|}{ Number of living children } \\
\hline None & 48 & 13.1 & 18 & 5.8 & \multirow{4}{*}{$\begin{array}{l}\chi^{2}=8.19 \\
p=.085\end{array}$} \\
\hline $1-2$ & 192 & 52.5 & 192 & 46.4 & \\
\hline $3-4$ & 99 & 27.0 & 138 & 33.3 & \\
\hline 5 or more & 27 & 7.3 & 60 & 14.3 & \\
\hline \multicolumn{6}{|l|}{ Use of the contraceptive method } \\
\hline No & 192 & 52.5 & 228 & 55.1 & \multirow{6}{*}{$\begin{aligned} \chi^{2} & =0.178 \\
p & =.862 \\
p & =.175 \\
\mathrm{FE} & =1.0 \\
{ }^{\mathrm{FE}} p & =.219 \\
\mathrm{FE}^{\mathrm{FE}} p & =.344\end{aligned}$} \\
\hline Pills & 45 & 12.3 & 48 & 11.6 & \\
\hline IUDs & 84 & 23.0 & 126 & 30.4 & \\
\hline Injections & 12 & 2.5 & 12 & 1.4 & \\
\hline Male physical barriers & 15 & 4.1 & 3 & 0.7 & \\
\hline Safe period & 18 & 2.5 & 3 & 0.7 & \\
\hline \multicolumn{6}{|c|}{ Effect of contraception on a sexual relation } \\
\hline No & 348 & 95.1 & 369 & 89.1 & $\chi^{2}=3.089$ \\
\hline Yes & 18 & 4.9 & 45 & 10.9 & $p=.079$ \\
\hline Circumcision & & & & & \\
\hline No & 232 & 63.4 & 246 & 59.4 & \\
\hline Simple & 98 & 26.8 & 93 & 22.5 & $\chi^{2}=0.372$ \\
\hline Moderate & 30 & 8.2 & 39 & 9.4 & ${ }^{\mathrm{MC}} p=.114$ \\
\hline Severe & 3 & 0.8 & 21 & 5.1 & \\
\hline Very severe & 3 & 0.8 & 15 & 3.6 & \\
\hline
\end{tabular}


Table 3. Distribution of the studied sample according to women's chronic disease, personal husband habits And sexual disorders $(\mathrm{n}=780)$

\begin{tabular}{|c|c|c|c|c|c|}
\hline \multirow{3}{*}{ Medical condition } & \multicolumn{4}{|c|}{ Studied women $(n=780)$} & \multirow{3}{*}{ Significance } \\
\hline & \multicolumn{2}{|c|}{$\begin{array}{l}\text { Women without sexual } \\
\text { dysfunction }(n=366)\end{array}$} & \multicolumn{2}{|c|}{$\begin{array}{l}\text { Women with sexual dysfunction } \\
(\mathrm{n}=414)\end{array}$} & \\
\hline & No. & $\%$ & No. & $\%$ & \\
\hline \multicolumn{6}{|l|}{ Suffer from a chronic disease } \\
\hline No & 330 & 90.2 & 336 & 81.2 & $\chi^{2}=4.207$ \\
\hline \#Yes & 36 & 9.8 & 78 & 18.8 & $p=.040^{*}$ \\
\hline Diabetes Mellitus & 6 & 1.6 & 30 & 7.2 & $p=.032 *$ \\
\hline Hypertension & 6 & 1.6 & 42 & 10.1 & $p=.004 *$ \\
\hline Goiter/thyroid disorder & 12 & 3.3 & 15 & 3.6 & ${ }^{\mathrm{FE}} p=1.0$ \\
\hline Anemia & 3 & 0.0 & 9 & 2.2 & ${ }^{\mathrm{FE}} p=.249$ \\
\hline Heart disease & 0 & 0.0 & 12 & 1.4 & ${ }^{\mathrm{FE}} p=.499$ \\
\hline Respiratory disease & 3 & 0.8 & 12 & 2.9 & ${ }^{\mathrm{FE}} p=.375$ \\
\hline Effect of disease on a sexual relation & {$[\mathrm{n}=36]$} & & {$[\mathrm{n}=78]$} & & \\
\hline No & 30 & 83.3 & 18 & 23.1 & $\chi^{2}=12.23$ \\
\hline Yes & 6 & 16.7 & 60 & 76.9 & $p=.0005^{*}$ \\
\hline Receives medication for the disease & {$[\mathrm{n}=36]$} & & {$[\mathrm{n}=78]$} & 0.0 & \\
\hline No & 0 & 0.0 & 6 & 7.7 & ${ }^{\mathrm{FE}} p=1.0$ \\
\hline Yes & 36 & 100.0 & 72 & 92.3 & \\
\hline Medication affects the sexual relation & {$[\mathrm{n}=36]$} & & {$[\mathrm{n}=72]$} & & \\
\hline No & 36 & 100.0 & 30 & 41.7 & ${ }^{\mathrm{FE}} p=.0007 *$ \\
\hline Yes & 0 & 0.0 & 42 & 58.3 & \\
\hline \multicolumn{6}{|l|}{ Inflammation of genital tract } \\
\hline No & 330 & 90.2 & 288 & 69.6 & $\chi^{2}=16.70$ \\
\hline Yes & 36 & 9.8 & 126 & 30.4 & $p=.0001^{*}$ \\
\hline Inflammation affects the sexual relation & {$[\mathrm{n}=36]$} & & {$[\mathrm{n}=126]$} & & \\
\hline No & 18 & 50.0 & 18 & 14.3 & ${ }^{\mathrm{FE}} p=.016^{*}$ \\
\hline Yes & 18 & 50.0 & 108 & 85.7 & \\
\hline \multicolumn{6}{|l|}{ Suffers stress } \\
\hline No & 273 & 74.6 & 195 & 47.1 & $\chi^{2}=20.387$ \\
\hline Yes & 93 & 25.4 & 219 & 52.9 & $p<.0001^{*}$ \\
\hline Stress affects the sexual relation & {$[\mathrm{n}=93]$} & & {$[\mathrm{n}=219]$} & & \\
\hline No & 27 & 29.1 & 18 & 8.2 & $\chi^{2}=7.640$ \\
\hline Yes & 66 & 70.9 & 201 & 91.8 & $p=.006^{*}$ \\
\hline \multicolumn{6}{|l|}{ \#Chronic illness of husband } \\
\hline Heart disease & 0 & 0.0 & 12 & 2.9 & ${ }^{\mathrm{FE}} p=.125$ \\
\hline Hypertension & 27 & 7.4 & 60 & 14.5 & $p=.069$ \\
\hline Diabetes & 42 & 11.5 & 51 & 12.3 & $p=.834$ \\
\hline Obesity & 18 & 7.4 & 42 & 10.1 & $p=.433$ \\
\hline Anemia & 3 & 0.8 & 6 & 1.4 & ${ }^{\mathrm{FE}} p=.602$ \\
\hline Hormonal disturbance & 0 & 0.0 & 3 & 0.7 & ${ }^{\mathrm{FE}} p=1.0$ \\
\hline Depression & 6 & 1.6 & 36 & 8.7 & $p=.012 *$ \\
\hline Others & 6 & 1.6 & 24 & 5.8 & ${ }^{\mathrm{FE}} p=.109$ \\
\hline \multicolumn{6}{|l|}{ \#Personal habits of husband } \\
\hline Heavy smoking & 105 & 28.7 & 174 & 42.0 & $p=.025^{*}$ \\
\hline Alcohol use & 6 & 1.6 & 3 & 0.7 & ${ }^{\mathrm{FE}} p=.602$ \\
\hline Hypnotic drugs & 3 & 0.8 & 45 & 10.9 & $p=.001 *$ \\
\hline Recreational drugs & 6 & 1.6 & 18 & 4.3 & ${ }^{\mathrm{FE}} p=.289$ \\
\hline \multicolumn{6}{|l|}{ \#Sexual disorders of husband } \\
\hline Low desire & 15 & 4.1 & 96 & 23.2 & $p<.0001^{*}$ \\
\hline Difficult erection & 9 & 2.5 & 48 & 11.6 & $p=.005^{*}$ \\
\hline Difficult keeping erection & 21 & 5.7 & 66 & 15.9 & $p=.009^{*}$ \\
\hline Premature ejaculation & 36 & 9.8 & 69 & 16.7 & $p=.107$ \\
\hline Inhibition or delayed ejaculation & 0 & 0.0 & 24 & 5.8 & ${ }^{\mathrm{FE}} p=.008^{*}$ \\
\hline Retrograde ejaculation & 0 & 0.0 & 21 & 5.1 & ${ }^{\mathrm{FE}} p=.016^{*}$ \\
\hline
\end{tabular}

\#Categories are not mutually exclusive; $\chi^{2}$ : Chi-Square test; $p$ : $p$-value of Chi-Square test; ${ }^{\mathrm{FE}} p$ : $p$-value for Fisher's Exact test; *significant at $p \leq .05$. 
Table 4. Relation between some characteristic of the studied women and sexual domains disorders $(\mathrm{n}=780)$

\begin{tabular}{|c|c|c|c|c|c|c|c|c|c|c|c|c|c|c|c|c|c|c|}
\hline \multirow[t]{2}{*}{ Characteristics } & \multicolumn{2}{|c|}{$\begin{array}{l}\text { DD } \\
(n=525)\end{array}$} & \multirow[t]{2}{*}{$\begin{array}{l}\text { OR } \\
(95 \% \text { CI }) \\
\end{array}$} & $\begin{array}{l}\mathrm{AD} \\
(\mathrm{n}=\end{array}$ & & $\begin{array}{l}\text { OR } \\
(95 \% \mathrm{CI})\end{array}$ & $\begin{array}{l}\text { LD } \\
(\mathrm{n}=\end{array}$ & & $\begin{array}{l}\text { OR } \\
(95 \% \mathrm{CI})\end{array}$ & $\begin{array}{l}\text { OD } \\
(\mathrm{n}=\end{array}$ & 399) & $\begin{array}{l}\text { OR } \\
(95 \% \mathrm{CI})\end{array}$ & $\begin{array}{l}\text { SD } \\
(\mathrm{n}=\end{array}$ & & $\begin{array}{l}\text { OR } \\
(95 \% \mathrm{CI})\end{array}$ & $\begin{array}{l}\text { PD } \\
(\mathbf{n}=\end{array}$ & & $\begin{array}{l}\text { OR } \\
(95 \% \text { CI })\end{array}$ \\
\hline & $\bar{n}$ & $\%$ & & $\mathrm{n}$ & $\%$ & & $\mathrm{n}$ & $\%$ & & $\mathbf{n}$ & $\%$ & & $\mathbf{n}$ & $\%$ & & $\mathbf{n}$ & $\%$ & \\
\hline Age (years) & & & & & & & & & & & & & & & & & & \\
\hline Less than 20 & 3 & 0.6 & $0.3(0.01-4.0)$ & 3 & 0.7 & $2.1(0.1-61.3)$ & 3 & 0.7 & $0.4(0.01-5.5)$ & 9 & 0.8 & $0.5(0.1-6.8)$ & 3 & 0.9 & $0.7(0.1-10.8)$ & 3 & 0.7 & $0.4(0.0-.8)$ \\
\hline $20-$ & 153 & 29.1 & 1 & 120 & 27.6 & 1 & 135 & 33.6 & 1 & 369 & 30.8 & 1 & 96 & 28.6 & 1 & 132 & 32.8 & 1 \\
\hline $30-$ & 222 & 42.3 & $0.9(0.5-1.7)$ & 171 & 39.3 & $0.9(0.5-1.6)$ & 129 & 32.1 & $0.4(0.2-0.8)^{*}$ & 441 & 36.8 & $0.6(0.3-1.2)$ & 129 & 38.4 & $0.8(0.4-1.5)$ & 147 & 36.6 & $0.5(0.3-0.0)$ \\
\hline $40-$ & 147 & 28.0 & $2.4(1.02-1.5)^{*}$ & 141 & 32.4 & $3.4(1.5-7.9)^{*}$ & 135 & 33.6 & $2.2(0.9-4.9)$ & 378 & 31.6 & $2.1(0.9-4.6)$ & 108 & 32.1 & $2.2(1.03-4.5)^{*}$ & 120 & 29.9 & $1.6(0.7-0.3)$ \\
\hline Educational leve & & & & & & & & & & & & & & & & & & \\
\hline Illiterate & 24 & 4.6 & $0.6(0.2-2.3)$ & 24 & 5.5 & $1.9(0.5-7.2)$ & 21 & 5.2 & $3.5(0.9-13.8)$ & 72 & 6.0 & $3.2(0.8-12.6)$ & 21 & 6.3 & $2.6(0.7-10.2)$ & 21 & 5.2 & $1.9(0.5-7.2)$ \\
\hline Read and write & 36 & 6.9 & $2.7(0.5-19.5)$ & 30 & 6.9 & $3.5(0.9-15.4)$ & 30 & 7.5 & $8.7(2.0-0.1)^{\circ}$ & 108 & 9.0 & $14.5(2.6-5.5)^{*}$ & 33 & 9.8 & $9.6(2.1-50.7)^{*}$ & 30 & 7.5 & $4.8(1.2-20.9)^{*}$ \\
\hline Secondary school & 201 & 38.3 & $0.9(0.4-1.9)$ & 195 & 44.8 & $2.6(1.3-5.3)^{*}$ & 201 & 50.0 & $6.8(3.1-5.4)^{*}$ & 504 & 42.1 & $3.0(1.4-6.4)^{*}$ & 156 & 46.4 & $2.8(1.3-5.9)^{*}$ & 180 & 44.8 & $2.8(1.4-5.8)^{*}$ \\
\hline University & 144 & 27.4 & $0.9(0.4-1.9)$ & 114 & 26.2 & $1.5(0.7-3.3)$ & 111 & 27.6 & $3.6(1.6-8.3)^{*}$ & 360 & 30.1 & $2.9(1.3-6.5)^{*}$ & 78 & 23.2 & $1.5(0.6-3.3)$ & 108 & 27.6 & $1.9(0.9-4.2)$ \\
\hline Postgraduate & 120 & 22.8 & 1 & 72 & 16.6 & 1 & 39 & 9.7 & 1 & 153 & 12.8 & 1 & 48 & 14.3 & 1 & 60 & 14.9 & 1 \\
\hline Occupation stat & & & & & & & & & & & & & & & & & & \\
\hline None & 171 & 32.6 & 1 & 54 & 37.2 & 1 & 56 & 41.8 & 1 & 156 & 39.0 & 1 & 141 & 42.0 & 1 & 159 & 39.6 & 1 \\
\hline Skilled & 18 & 3.4 & $3.2(0.4-72.9)$ & 5 & 3.5 & $1.5(0.2-12.1)$ & 5 & 3.7 & $1.4(0.2-11.0)$ & 15 & 3.8 & $1.7(0.3-13.4)$ & 18 & 5.4 & $5.1(0.6-117.4)$ & 12 & 3.0 & $0.9(0.2-5.2)$ \\
\hline Semiskilled & 51 & 9.7 & $1.5(0.5-4.8)$ & 15 & 10.3 & $1.2(0.4-3.3)$ & 15 & 11.3 & $1.0(0.4-3.0)$ & 42 & 10.5 & $1.1(0.4-2.9)$ & 45 & 13.3 & $1.6(0.6-4.6)$ & 33 & 8.2 & $0.6(0.2-1.6)$ \\
\hline Professional & 270 & 51.4 & $1.1(0.6-1.9)$ & 69 & 47.6 & $0.7(0.4-1.2)$ & 55 & 41.0 & $0.4(0.2-0.7)^{*}$ & 177 & 44.4 & $0.5(0.3-0.9)^{*}$ & 129 & 38.4 & $0.4(0.2-0.7)^{*}$ & 177 & 44.0 & $0.5(0.3-0.9)^{*}$ \\
\hline Student & 15 & 2.9 & $0.7(0.1-3.2)$ & 2 & 1.4 & $0.2(0.1-1.0)$ & 3 & 2.2 & $0.3(0.1-1.4)$ & 9 & 2.3 & $0.3(0.1-1.7)$ & 3 & 0.9 & $0.1(0.0-0.9)^{*}$ & 21 & 5.2 & $2.3(0.4-16.7)$ \\
\hline BMI & & & & & & & & & & & & & & & & & & \\
\hline Underweight & 3 & 0.6 & UN & 1 & 0.7 & UN & 1 & 0.7 & UN & 3 & 0.8 & UN & 3 & 0.9 & UN & 3 & 0.7 & UN \\
\hline Normal & 141 & 26.8 & 1 & 37 & 25.5 & 1 & 36 & 26.9 & 1 & 99 & 24.8 & 1 & 84 & 25.0 & 1 & 99 & 24.6 & 1 \\
\hline Overweight & 168 & 32.0 & $0.9(0.5-1.9)$ & 47 & 32.4 & $1.1(0.6-2.2)$ & 40 & 29.9 & $0.9(0.4-1.7)$ & 129 & 32.3 & $1.2(0.6-2.3)$ & 114 & 33.9 & $1.2(0.6-2.4)$ & 132 & 32.9 & $1.2(0.6-2.4)$ \\
\hline Obese & 213 & 40.6 & $1.3(0.7-2.6)$ & 60 & 41.4 & $1.4(0.7-2.7)$ & 57 & 42.5 & $1.3(0.7-2.6)$ & 168 & 42.1 & $1.5(0.8-2.9)$ & 135 & 40.2 & $0.3(0.7-2.5)$ & 168 & 41.8 & $1.5(0.8-2.9)$ \\
\hline Practice exercise & & & & & & & & & & & & & & & & & & \\
\hline Daily & 15 & 2.9 & 1 & 4 & 2.8 & 1 & 2 & 1.5 & 1 & 12 & 3.0 & 1 & 9 & 2.7 & 1 & 18 & 2.2 & 1 \\
\hline Several times/wk & 42 & 8.0 & $3.4(0.6-22.1)$ & 8 & 5.5 & $1.3(0.2-7.7)$ & 8 & 6.0 & $3.3(0.4-29.5)$ & 30 & 7.5 & $1.9(0.3-11.8)$ & 30 & 8.9 & $2.9(0.5-20.3)$ & 39 & 9.7 & $5.8(0.9-42.9)$ \\
\hline Once/wk & 51 & 9.7 & $1.9(0.4-9.5)$ & 13 & 9.0 & $1.5(0.3-8.1)$ & 12 & 9.0 & $3.4(0.5-27.7)$ & 18 & 6.8 & $0.8(0.2-4.5)$ & 21 & 6.3 & $0.9(0.2-5.7)$ & 36 & 9.0 & $2.0(0.4-12.2)$ \\
\hline Rarely & 163 & 32.0 & $3.2(0.8-13.9)$ & 43 & 29.6 & $2.2(0.5-9.9)$ & 42 & 31.3 & $5.4(0.9-38.9)$ & 123 & 30.8 & $1.9(0.5-8.9)$ & 84 & 25.0 & $1.5(0.3-9.7)$ & 123 & 30.6 & $3.1(0.7-15.8)$ \\
\hline Never & 249 & 47.4 & $2.4(0.6-9.6)$ & 77 & 53.1 & $2.8(0.7-12.2)$ & 70 & 52.2 & $5.7(1.1-40.1)^{*}$ & 207 & 51.9 & $2.2(0.5-9.3)$ & 192 & 57.1 & $2.8(0.6-14.0)$ & 195 & 48.5 & $2.9(0.7-14.5)$ \\
\hline Parity & & & & & & & & & & & & & & & & & & \\
\hline None & 45 & 8.6 & 1 & 30 & 6.9 & 1 & 36 & 9.0 & 1 & 24 & 6.0 & 1 & 18 & 5.4 & 1 & 36 & 9.0 & 1 \\
\hline $1-2$ & 234 & 44.6 & $1.3(0.5-3.3)$ & 189 & 43.4 & $1.7(0.7-4.4)$ & 159 & 39.6 & $0.9(0.4-2.2)$ & 48 & 45.9 & $2.2(0.8-6.0)$ & 153 & 45.5 & $2.4(0.8-7.1)$ & 186 & 46.3 & $1.2(0.5-3.0)$ \\
\hline $3-4$ & 177 & 33.7 & $3.5(1.3-9.7)^{*}$ & 150 & 34.5 & $2.5(0.9-6.8)$ & 144 & 35.8 & $1.7(0.6-4.4)$ & 132 & 33.1 & $2.6(0.9-7.4)$ & 117 & 34.8 & $3.0(1.0-9.5)^{*}$ & 120 & 29.9 & $1.1(0.4-2.9)$ \\
\hline $5-6$ & 48 & 9.1 & $2.4(0.6-10.2)$ & 42 & 9.7 & $3.2(0.8-12.9)$ & 45 & 11.2 & $2.9(0.7-11.9)$ & 42 & 10.5 & $4.5(1.1-8.9)^{*}$ & 33 & 9.8 & $3.7(0.9-15.7)$ & 39 & 9.7 & $1.9(0.5-7.3)$ \\
\hline 7 or more & 21 & 4.0 & $5.1(0.5-127.9)$ & 24 & 5.5 & UN & 18 & 4.4 & $3.5(0.5-31.2)$ & 18 & 4.5 & $6.8(0.9-63.0)$ & 15 & 4.5 & $5.6(0.8-43.6)$ & 21 & 5.1 & $8.2(0.8-203.4)$ \\
\hline Gravidity & & & & & & & & & & & & & & & & & & \\
\hline None & 42 & 8.0 & 1 & 27 & 6.2 & 1 & 33 & 8.2 & 1 & 21 & 5.3 & 1 & 15 & 4.5 & 1 & 33 & 8.2 & 1 \\
\hline $1-2$ & 189 & 36.0 & $0.9(0.3-2.6)$ & 159 & 36.6 & $1.6(0.6-4.4)$ & 129 & 32.1 & $0.7(0.3-2.0)$ & 147 & 36.8 & $1.9(0.7-5.9)$ & 126 & 37.5 & $2.4(0.8-8.1)$ & 153 & 38.1 & $1.0(0.4-2.8)$ \\
\hline $3-4$ & 177 & 33.7 & $1.2(0.4-3.5)$ & 141 & 32.4 & $1.7(0.6-4.9)$ & 135 & 33.6 & $1.1(0.4-3.0)$ & 135 & 33.8 & $2.3(0.8-6.9)$ & 114 & 33.9 & $2.6(0.8-9.1)$ & 120 & 29.8 & $0.9(0.3-2.4)$ \\
\hline $5-6$ & 72 & 13.7 & $2.3(0.6-9.6)$ & 63 & 14.5 & $3.4(0.9-12.7)$ & 66 & 16.4 & $2.8(0.7-10.5)$ & 57 & 14.3 & $3.7(1.0-14.2)$ & 57 & 17.0 & $5.9(1.5-24.9)^{*}$ & 57 & 14.2 & $1.7(0.5-6.2)$ \\
\hline 7 or more & 45 & 8.6 & $2.1(0.4-11.0)$ & 45 & 10.3 & $5.4(1.1-28.2)^{*}$ & 39 & 9.7 & $2.2(0.5-9.5)$ & 39 & 9.8 & $1.0(0.2-4.6)$ & 24 & 7.1 & $2.5(0.5-11.9)$ & 39 & 9.7 & $2.2(0.5-9.5)$ \\
\hline Abortion & & & & & & & & & & & & & & & & & & \\
\hline None & 354 & 67.4 & 1 & 282 & 64.8 & 1 & 255 & 63.5 & 1 & 261 & 65.4 & 1 & 228 & 67.8 & 1 & 267 & 66.4 & 1 \\
\hline $1-2$ & 147 & 28.0 & $1.1(0.6-2.0)$ & 132 & 30.4 & $1.4(0.8-2.6)$ & 126 & 31.3 & $1.5(0.9-2.8)$ & 120 & 30.1 & $1.3(0.7-2.4)$ & 96 & 28.6 & $1.1(0.6-1.9)$ & 120 & 29.9 & $1.3(0.7-2.3)$ \\
\hline $3-$ & 24 & 4.6 & $2.0(0.4-14.3)$ & 21 & 4.8 & $2.1(0.5-10.6)$ & 21 & 5.2 & $2.6(0.6-12.9)$ & 18 & 4.5 & $1.6(0.4-6.9)$ & 12 & 3.6 & $0.9(0.2-3.8)$ & 15 & 3.7 & $1.0(0.2-4.2)$ \\
\hline No. of children & & & & & & & & & & & & & & & & & & \\
\hline None & 42 & 8.0 & 1 & 27 & 6.2 & 1 & 33 & 8.2 & 1 & 21 & 5.3 & 1 & 15 & 4.5 & 1 & 33 & 8.2 & 1 \\
\hline $1-2$ & 246 & 46.9 & $1.3(0.5-3.4)$ & 201 & 46.2 & $1.8(0.7-4.9)$ & 171 & 42.5 & $0.9(0.4-2.5)$ & 192 & 48.1 & $2.4(0.9-6.9)$ & 162 & 48.2 & $2.8(0.9-9.1)$ & 195 & 48.5 & $1.2(0.5-3.2)$ \\
\hline $3-4$ & 168 & 32.0 & $1.7(0.6-4.9)$ & 141 & 32.4 & $2.5(0.9-6.9)$ & 135 & 33.6 & $1.6(0.6-4.3)$ & 126 & 31.6 & $2.8(0.9-8.3)$ & 114 & 33.9 & $3.5(1.1-12.1)^{*}$ & 117 & 29.1 & $1.2(0.4-3.2)$ \\
\hline $5-6$ & 48 & 9.1 & $2.3(0.5-10.2)$ & 42 & 9.7 & $3.3(0.8-13.9)$ & 45 & 11.2 & $2.9(0.7-12.5)$ & 42 & 10.5 & $4.9(1.2-21.4)^{*}$ & 30 & 8.9 & $3.5(0.8-15.8)$ & 36 & 9.0 & $1.6(0.4-6.1)$ \\
\hline 7 or more & 21 & 4.0 & $5.0(0.5-126.1)$ & 24 & 5.5 & UN & 18 & 4.5 & $3.6(0.5-32.2)$ & 18 & 4.5 & $7.3(0.9-70.4)$ & 15 & 4.5 & $6.3(0.9-53.4)$ & 21 & 5.2 & $8.3(0.8-208.5)$ \\
\hline Age of youngest & & & & & & & & & & & & & & & & & & \\
\hline Less than 1 year & 99 & 18.9 & $0.2(0.1-0.6)^{*}$ & 81 & 18.6 & $0.1(0.1-0.4)^{*}$ & 78 & 19.4 & $0.2(0.1-.6)^{*}$ & 72 & 18.0 & $0.2(0.1-0.6)^{*}$ & 66 & 19.6 & $0.3(0.1-0.7)^{*}$ & 84 & 20.9 & $0.5(0.2-1.1)$ \\
\hline $1-<5$ & 228 & 43.4 & $0.2(0.1-0.7)^{*}$ & 180 & 41.4 & $0.1(0.1-0.4)^{*}$ & 162 & 40.3 & $0.2(0.1-.5)^{*}$ & 162 & 40.6 & $0.3(0.1-0.6)^{*}$ & 132 & 39.3 & $0.2(0.1-0.5)^{*}$ & 174 & 43.3 & $0.4(0.2-0.9)^{*}$ \\
\hline $5-<10$ & 87 & 16.6 & $0.3(0.1-1.2)$ & 63 & 14.5 & $0.1(0.1-0.5)^{*}$ & 60 & 14.9 & $4.1(1.4-.3)^{*}$ & 69 & 17.3 & $0.4(0.1-1.1)$ & 48 & 14.3 & $0.3(0.1-0.7)^{*}$ & 57 & 14.2 & $0.4(0.1-1.0)$ \\
\hline $10-$ & 111 & 21.1 & 1 & 111 & 25.5 & 1 & 102 & 25.4 & 1 & 96 & 24.1 & 1 & 90 & 26.8 & 1 & 87 & 21.6 & 1 \\
\hline Type of delivery & & & & & & & & & & & & & & & & & & \\
\hline Vaginal delivery & 252 & 48.0 & $2.5(1.4-4.6)^{*}$ & 213 & 49.0 & $2.1(1.2-3.6)^{*}$ & 192 & 47.8 & $1.8(1.04-3.0)^{*}$ & 207 & 51.9 & $2.5(1.5-4.4)^{*}$ & 156 & 46.4 & $1.5(0.9-2.5)$ & 186 & 46.3 & $1.6(0.9-2.6)$ \\
\hline Cesarean section & 282 & 53.7 & $0.6(0.3-1.1)$ & 234 & 53.7 & $0.7(0.4-1.2)$ & 222 & 55.2 & $0.8(0.5-1.4)$ & 219 & 54.9 & $0.8(0.5-1.3)$ & 192 & 57.1 & $0.9(0.6-1.6)$ & 228 & 56.7 & $0.9(0.6-1.6)$ \\
\hline Episiotomy & 144 & 27.3 & $1.8(0.9-3.6)$ & 123 & 28.3 & $1.7(0.9-3.1)$ & 117 & 29.1 & $1.7(0.9-3.3)$ & 114 & 28.6 & $1.6(0.9-3.0)$ & 78 & 23.2 & $0.9(0.5-1.7)$ & 105 & 26.1 & $1.2(0.7-2.3)$ \\
\hline Suffer from chro & ic dise & & & & & & & & & & & & & & & & & \\
\hline No & 423 & 80.6 & 1 & 348 & 80.0 & 1 & 318 & 79.1 & 1 & 315 & 78.9 & 1 & 276 & 82.1 & 1 & 330 & 82.1 & 1 \\
\hline Yes & 102 & 19.4 & $4.9(1.6-16.9)^{*}$ & 87 & 20.0 & $2.9(1.3-7.1)^{*}$ & 84 & 20.9 & $3.1(1.4-.1)^{*}$ & 84 & 21.1 & $3.1(1.4-7.3)^{*}$ & 60 & 17.9 & $1.6(0.8-3.3)$ & 72 & 17.9 & $1.8(0.8-3.8)$ \\
\hline Receives medica & & & & & & & & & & & & & & & & & & \\
\hline No & 429 & 81.7 & 1 & 354 & 81.4 & 1 & 324 & 80.6 & 1 & 321 & 80.5 & 1 & 279 & 83.0 & 1 & 333 & 82.8 & 1 \\
\hline Yes & 96 & 18.3 & $4.5(1.5-15.7)^{*}$ & 81 & 18.6 & $2.7(1.2-6.5)^{*}$ & 78 & 19.4 & $2.8(1.2-6.5)^{*}$ & 78 & 19.5 & $2.8(1.2-6.7)^{*}$ & 57 & 17.0 & $1.6(0.7-3.4)$ & 69 & 17.2 & $1.8(0.8-3.9)$ \\
\hline Inflammation of & enital & & & & & & & & & & & & & & & & & \\
\hline No & 393 & 74.9 & 1 & 315 & 72.4 & 1 & 276 & 68.7 & 1 & 270 & 67.7 & 1 & 246 & 73.2 & 1 & 282 & 70.1 & 1 \\
\hline Yes & 132 & 25.1 & $4.4(3.5-17.4)^{*}$ & 120 & 27.6 & $2.8(1.4-5.7)^{*}$ & 126 & 31.3 & $4.3(2.1-9.3)^{*}$ & 129 & 32.3 & $5.0(2.4-11.0)^{*}$ & 90 & 26.8 & $1.9(0.9-3.6)$ & 120 & 29.9 & $3.4(1.7-7.0)^{*}$ \\
\hline Suffers stress & & & & & & & & & & & & & & & & & & \\
\hline No & 276 & 52.6 & 1 & 210 & 48.3 & 1 & 174 & 43.3 & 1 & 180 & 45.1 & 1 & 165 & 49.1 & 1 & 204 & 50.7 & 1 \\
\hline Yes & 249 & 47.4 & $2.8(1.5-5.1)^{*}$ & 225 & 51.7 & $3.2(1.8-5.6)^{*}$ & 228 & 56.7 & $4.6(2.6-8.2)^{*}$ & 219 & 54.9 & $3.8(2.2-6.6)^{*}$ & 171 & 50.9 & $2.2(1.3-3.8)^{*}$ & 198 & 49.3 & $2.3(1.3-3.9)^{*}$ \\
\hline
\end{tabular}


Among the studied subjects, Sexual dysfunction was observed to be most frequent in domains of sexual desire $(67.3 \%)$, followed by sexual arousal (55.8\% )which is consistent with what is reported in the literature, ${ }^{[14,39,40]}$ but contrary to other studies findings. ${ }^{[33,35,41,42]}$ This variance could be due to differences in cut-off points determined for FSD domains. It is evident from the present result that several women can experience adequate sexual satisfaction with their sexual relation, despite an inability to achieve arousal or orgasm. This may be attributed to misconceptions about sex, negative emotion, difficult communication and relationship between couples and low level of education which lead to a reduced sexual technique. Additionally, there was a strong relation between lubrication disorders and experiencing pain during sexual intercourse, as both of which is the same percentage $(51.5 \%)$. This result is reasonable because inadequate vaginal lubrication is a significant cause of coital pain.

In the literature, Socio-demographic characteristics of the woman and her husband were found to be strongly prognosis of FSD. FSD has been reported to be more prevalent among participants who are older. ${ }^{[14,43,44]}$ As the older women can take a long time to reach sexual stimulation, vaginal lubrication, and orgasm as well as decrease the level of orgasm which results from the aging physiological and anatomical changes. The present study result is in accordance with the literature but the contrast to other studies results. ${ }^{[37,45,46]}$ The women level of education is a significant variable in awareness and construing the sexual issues.

Further that, it helps women to acquire more information about female sexual acts from different media and understand the sexual needs of women. In the present study, FSD has been reported to be more prevalent among participants who have lower levels of education. This result is similar to other study results, ${ }^{[22,45,47]}$ but contrarily to other studies results. ${ }^{[43,48]}$ With regard to having a job, non-job status was significantly high among sexual dysfunction women while professional work was high among women without sexual dysfunction. This result is anticipated because there is a close relationship between the level of education and professional work. Women who have a university or higher education level can get professional work, unlike the lower levels of education. This result is in line with other studies results. ${ }^{[21,22,33]}$ Other study failed to get association. ${ }^{[19,45]}$ In the present study, husband older age and lower educated were significant risk factors for FSD; this result correlates with other studies results. ${ }^{[10,35]}$ One of the main reasons for this is that the lower educated husbands do not have enough sex education, have wrong beliefs regard sexual act, do not have enough knowledge about ways of sexual excitement and stimulation to help women to reach orgasm which results in reduced sexual performance and technique. In contrast, it was reported that husbands' age did not emerge significantly in logistic regression as contributory of FSD. ${ }^{[19]}$

The present study revealed that traditional type of marriage was significantly associated with FSD while marriage based on love story more prevalent among female without SD. For that, a harmonious marital life based on love story may allow men and women to have a happy and harmonious marriage and to reach a sexual concordance and satisfaction. On the contrary, Reda et al. ${ }^{[49]}$ said that marriage whether love or arranged, were not significantly associated with the FSD. It was mentioned that there was a comparable relationship between sexual intercourse rate and sexual desire and that these were affecting the sexual satisfaction. ${ }^{[43]}$ A fact that correlates with the findings of the present study as the women with SD have lower sexual intercourse rate. As for body mass index (BMI), no statistically significant difference was found between the two groups regarding their body mass index whereas the most women of both groups were overweight and obese. This result is accordance with previous other studies results ${ }^{[37,46,47,50]}$ while it is a contrast to other. ${ }^{[51]}$

The differences in the menstrual, obstetric parameters and number of living children among female without sexual dysfunction and dysfunctional sexual women were statistically insignificant. This result is similar to other study results, ${ }^{[10,19]}$ while contrary to other studies findings. ${ }^{[22,43,45]}$ In additionally, female circumcision was found to be not associated with FSD in the present study which less than two-thirds of women in the two groups have no circumcision and about one-fourth of circumcised women in two groups have a simple degree of circumcision. The decline of female circumcision prevalence in this study pointed to the country's efforts to attack such practice by increasing people's awareness and changing their attitudes toward that habit. This finding duplicates those of previous Egyptian studies. ${ }^{[49,52,53]}$ However, contrary to other papers in Egypt. ${ }^{[10,35]}$ Vaginal delivery and episiotomy were highly significant variables with female sexual dysfunction, this in line with other studies results. ${ }^{[8,35,54,55]}$ In the present study, the type of contraceptive used was not statistically significantly associated with sexual dysfunction which is similar to Amirkhani et al. ${ }^{[50]}$ but in contrast to Tekin et al. ${ }^{[22]}$

Medical risk factors were investigated in all women, and the findings were compared between the women with and without SD. It can show from the present study that diabetes mellitus, hypertension, and thyroid disorder were significantly correlated with female sexual dysfunction. It was confirmed that metabolic syndrome, hyperlipidemia, hypertension, dia- 
betes, and coronary heart disease are associated with more FSD and impaired desire. Diabetes and hypertension may affect pelvic and clitoral blood flow after sexual stimulation leading to reduced blood flow, engorgement, lubrication and orgasm. ${ }^{[56-58]}$ Also, receiving medication for chronic diseases was significantly correlated with FSD, which is similar to Esfehani et al. ${ }^{[39]}$ Inflammation of the genital tract was also considered a significant variable for sexual dysfunction in the present study, which is in contrast to Afefy. ${ }^{[59]}$ In the present study, stress is significantly correlated with female sexual dysfunction, $p=.006$ which in line with previous studies results which established that daily life stressors are connected with self-reported sexual problems and lower sexual satisfaction in women. In addition, studies examining cortisol levels as an indicator of stress have shown that higher levels of cortisol are significantly associated with poorer selfreported sexual functioning and less genital reaction during sexual arousal. ${ }^{[60,61]}$

Various medical problems of the husband, as well as personal habits, appear to affect the sexual function of women. The present study reveals that husbands suffer from depression were significantly correlated with the extent of female sexual problems, as the husband were heavy smokers and used hypnotic drugs. In the literature, the relevant data are investigated. Multivariate analysis mentioned that hypertension $(\mathrm{OR}=5.4)$, diabetes mellitus ( $\mathrm{OR}=5.4$ ) and smoking ( $\mathrm{OR}$ $=3.1$ ) were significant risk factors for erectile dysfunction (ED) ${ }^{[62]}$ Also, Battaglia et al. ${ }^{[63]}$ mentioned that cigarette smoking could decrease central and peripheral tissue perfusion and increase the risks of genital vessel stiffness and impaired sexual performance and cause an erectile disorder. Furthermore, Johannes et al. ${ }^{[64]}$ stated that depression is significantly associated with self-reported sexual functioning as approximately $40 \%$ of sexual arousal disorders participant reported having concurrent depression.

In the literature, a husband with sexual problems and who did not share sexuality with their spouses had significantly higher sexual dysfunction rate. ${ }^{[43]}$ The present study results are in line with the literature in which low desire, difficult erection, difficult keeping erection, inhibition or delayed ejaculation and retrograde ejaculation was significantly high among husbands of women with SD. It was reported that male sexual dysfunction as premature ejaculation and erectile dysfunction was associated with the reduction of libido or loss of sexual desire in the female partner as well as with reduced sexual satisfaction which considered a risk factor for FSD. ${ }^{[35,65]}$ Bukhari et al. ${ }^{[66]}$ mentioned that premature ejaculation (PE) causes loss of sexual satisfaction among couples which lead to the termination of relationships. Other study failed to find a significant because of small sample size. ${ }^{[49]}$

According to the results of the multiple logistic regression analysis were achieved from the final model for each sexual domain. Disorder of women sexual domains was significantly correlated with increasing age of women and low level of education as well as professional work. This result is consistent with the previous studies results..$^{[10,14,19,52]}$ From the researcher's point of view regarding professional work and its impact on sex due to the emotional stress and physical diseases it causes and thus destroys the woman's psychological, physical and sexual health. It was mentioned that sexual domains were significantly associated with BMI particularly, desire, arousal, and satisfaction domains. ${ }^{[52]}$ The present study result is not consistent with the previous study result.

Furthermore, the present study revealed that increasing numbers of parity and pregnancies, as well as vaginal deliveries, were significantly correlated with the extent of most sexual domain disorders. It is possibly due to anatomical changes, weakening of the pelvic floor muscles and ligaments, and maybe the presence of a different degree of genital tract prolapse and piles associated with repeated pregnancies and vaginal deliveries and accompanying complications. In the literature, the relevant data are different. ${ }^{[67,68]}$ This is in addition to the impact of increasing the number of children and their young age on a sexual domain, although older ages also have an impact. Ghanbarzadeh et al. ${ }^{[67]}$ mentioned that unsatisfactory sexual activity was positively associated with having a teenager and elder children and for their respect while they are staying at home (OR 9.24, 95\% CI 4.44-19.23). Finally, stress was significantly aggravating factor for all sexual domain disorders as shown in the present study result. This explains physiologically that stress inhibits the sexual activity by creating cognitive and emotional changes which decrease the individual concentrate on sexual relationship and excitement. ${ }^{[17]}$

The strength of the study: the first strength is the sample of the study was collected randomly from several healthcare facilities such as hospitals and MCH centers in Damietta governorate that can be generalized to the entire community as well. The second strength is the study role of the husband in the study which can have an essential part in women sexual life. The third strength is the using of Female sexual function index (FSFI) Arabic version by Anis et al. (2011) ${ }^{[31]}$ to address sexual disorders which are an integral part of the compilation of data.

Limitations of this study were first concerning the importance and privacy of the question; it was difficult for some women to express their sexual problems, as well as the questions that were answered faced some obscurities. So, the researcher 
tried to solve this problem by protecting the confidentiality of the participants, and the necessary cases were referred to specific health specialty. Another possible limitation would be the potential under-reporting of female-related or malerelated data as a result of shame. All efforts were made to get as much complete data as possible.

\section{Conclusion}

The present study results concluded that female sexual dysfunction is a significant health problem that affected more than half of the studied women. Some maternal factors were significantly correlated with the extent of female sexual problems; age group, job, level of education, mode of delivery and medical condition. Furthermore, husband factors have a significant correlation with lower FSI score, especially female sexual problems.

\section{Recommendation}

From the present study results, the following recommendations are suggested:

- Continuing educational program should be imple- mented and developed to teach the nurses at maternal and child health centers and clinics about female sexual dysfunction as a serious, neglected and hidden problem.

- Future studies are required to assess the knowledge and awareness of both female and male regarding their genitals organs and sexual issues urgently needed.

- Future studies are required to assess the knowledge; awareness and competency of nurses regarding female sexual issues urgently needed.

- All health facilities should be equipped with educational and health teaching materials about sexual issues, and they should be written simply with illustrations to suit all educational levels for both sexes.

- Bay attention to the medical and surgical aspects of women as the aggravating factors for sexual problems as well as has a negative impact on their quality of life.

\section{CONFLiCTS OF INTEREST Disclosure}

The authors declare that there is no conflict of interest.

\section{REFERENCES}

[1] Movahed M, Azizi T. A Survey of the Relationship between Women's Sexual Satisfaction and Spouses' Conflicts. Women in Development \& Politics Research on Women. 2011; 9: 191-216.

[2] Murtagh J. Female sexual function, dysfunction, and pregnancy: Implications for practice. J Midwifery Women's Health. 2010; 55(5): 438-46. PMid:20732665 https://doi.org/10.1016/j.jmwh.2 009.12 .006

[3] Jamali S, Mosalanejad L. Sexual dysfunction in Iranian pregnant women. Ira J Med Reprod. 2013; 11(6): 479-86.

[4] Darwish M, Hamza W, Aziz M, et al. Sexual and Reproductive Health Experiences of Married Adolescent Girls in Rural Upper Egypt. In: Abdel-Tawab N., Saher, S., \& El Nawaawi, N., (editors). "Breaking the Silence: Learning about Youth Sexual and Reproductive Health in Egypt." Cairo: Population Council. 2013.

[5] Bhavsar V. Cultural factors and sexual dysfunction in clinical practice. Advances in Psychiatric Treatment. 2013; 19: 144-152. https: //doi.org/10.1192/apt.bp.111.009852

[6] Roudi-Fahimi F, El Feki SH. Facts of Life: Youth Sexuality and Reproductive Health in the Middle East and North Africa. Population Reference Bureau. 2015.

[7] Amidu N, Owiredu W, Woode E, et al. Prevalence of male sexual dysfunction among Ghanaian populace: myth or reality? International Journal of Impotence Research. 2010; 22: 337-42.

[8] Lou WJ, Chen B, Zhu L. Prevalence and Factors Associated with Female Sexual Dysfunction in Beijing, China. Chinese Medical Journal 2017; 130: 1389-1394. PMid:28584199 https ://doi .org/10.4 103/0366-6999. 207466

[9] Moore CK. Female sexual function and dysfunction Scott. Tenth Edition. In: McDougal W, Wein AJ, Kavoussi LR, Novick AC, Partin
AW, Peters CA, et al., editors. Campbell-Walsh Urology, 6. Philadelphia: Elsevier: Saunders; 2010. 823-33.

[10] Hassanin IM, Helmy YA, Fathalla MM, et al. Prevalence and characteristics of female sexual dysfunction in a sample of women from upper Egypt. Int. J. Gynecol. Obstet. 2010; 108: 219-23.

[11] Mazinani, R. A.M.M., Kaskian, A., Kashanian M. Evaluation of the prevalence of sexual dysfunctions and its related factors in women. Razi Journal of Medical Sciences. 2013; 19(105): 60-8.

[12] Zhang A, Pan L, Chen X, et al. An investigation of female sexual dysfunction among urban Chinese women in Nanjing. Zhonghua Nan Ke Xue. 2011; 17(6): 488-491.

[13] Zhang H, Yip P. Female sexual dysfunction among young and middleaged women in Hong Kong: prevalence and risk factors. The Journal of Sexual Medicine. 2012.

[14] Ishak I, Low W, Othman S. Prevalence, risk factors, and predictors of female sexual dysfunction in a primary care setting: a survey found. The Journal of Sexual Medicine. 2010; 7(9).

[15] Shaeer O, Shaeer K, Shaeer E. The global online sexuality survey (GOSS): female sexual dysfunction among4 internet users in the reproductive age group in the Middle East. The Journal of Sexual Medicine. 2012; 9(2).

[16] Rouzi1 AA, Sahly N, Sawan D, et al. The prevalence of sexual dysfunction in the female healthcare providers in Jeddah, Saudi Arabia. SCIENTIFIC REPORTS. 2013

[17] Hamilton LD, Meston CM. Chronic stress and sexual function in women. The Journal of Sexual Medicine. 2013; 10(10): 2443-54. PMid:23841462 https ://doi.org/10.1111/jsm. 12249

[18] American Psychiatric Association. Manual diagnostic statistics de transtornos mentais: DSM-5. 2014.

[19] Balon R, Woodard TL. Diagnosis of Female Sexual Dysfunction, Management of Sexual Dysfunction in Men and Women, Department 
of Psychiatry and Gynecologic Oncology and Reproductive. 2012; 32: 473-479.

[20] Anderson JL. Acknowledging Female Sexual Dysfunction in Women with Cancer. Clinical Journal of Oncology Nursing. 2013; 17: 233235. PMid:23715699 https://doi.org/10.1188/13.CJON. 23 3-235

[21] Rizk HII, Gaafar HM. Sexual Problems among Young Married Women Attending Primary Health Care Facilities in Cairo, Egypt: An Exploratory Study. Middle-East Journal of Scientific Research. 2013; 23(11): 2763-2772.

[22] Tekin BY, Ural UM, Ustuner I, et al. Evaluation of Female Sexual Function Index and Associated Factors among Married Women in North Eastern Black Sea Region of Turkey. Turkish Journal of Obstetrics and Gynecology. 2014; 11: 153-158. PMid:28913009 https://doi.org/10.4274/tjod.43815

[23] Tsai TF, Yeh CH, Hwang TIS. Female Sexual Dysfunction: Physiology, Epidemiology, Classification, Evaluation, and Treatment. Urol Sci. 2011; 22(1): 7-13. https://doi .org/10.1016/S1879-522 6(11) $60002-\mathrm{X}$

[24] Rowland D, Keeney C, Slob A. Sexual response in men with inhibited or retarded ejaculation. International Journal of Impotence Research. 2014; 16: 270-74. PMid:14961061 https://doi.org/10.1038/ sj.ijir.3901156

[25] Althof S. Sex therapy in the age of pharmacotherapy. Annual Review of Sex Research. 2006. 116-32.

[26] McCabe MP. Institute for Health and Ageing, Australian Catholic University, Melbourne, Australia Springer Science Business Media New York 2016 307. In: Lipshultz, L.I. et al., Eds., Management of Sexual Dysfunction in Men and Women, University of Piraeus, Piraeus. 2016.

[27] Costa LHR, Coelho EAC. Sexuality and the intersection with caring in the nurse's professional practice. Rev Bras Enferm. 2013; 66(4): 493-500. PMid:24008701

[28] Mamdouh HM, Abdou AM, Kharboush I, et al. Prevalence and characteristics of female sexual dysfunction among a sample of Egyptian women. American Journal of Research Communication. 2017; 5(5): 62-74.

[29] Park K. Textbook of Preventive and Social Medicine. 20th ed. Banarsidas Bhanot Publishers Jabalpur. 2010; 347.

[30] Rosen R, Brown C, Heiman J, et al. The Female Sexual Function Index (FSFI): a multidimensional self-report instrument for the assessment of female sexual function. J Sex Marital Ther. 2000; 26: 191208. PMid:10782451 https://doi.org/10.1080/0092623002 78597

[31] Anis TH, Gheit SA, Saied HS, et al. Arabic translation of the female sexual function index and validation in an Egyptian population. J Sex Med. 2011; 8(12): 3370-3378. PMid:21995610 https://doi.org/10.1111/j.1743-6109.2011.02471.x

[32] Tavakol M, Dennick R. Making Sense of Cronbach's Alpha. International Journal of Medical Education. 2011; 2: 53-55. PMid:28029643 https://doi.org/10.5116/ijme.4dfb.8dfd

[33] El-Tahlawi1 S, Mohammad NE, Elsary AY, et al. Female Sexual Dysfunction in Elfayoum Governorate. Advances in Sexual Medicine. 2018; 8: 1-13. https://doi.org/10.4236/asm. 2018.81001

[34] Giraldi A, Rellini A, Pfaus JG, et al. Questionnaires for assessment of female sexual dysfunction: a review and proposal for a standardized screener. J Sex Med. 2011; 8: 2681-2706. PMid:21810182 https://doi.org/10.1111/j.1743-6109.2011.02395.x

[35] Ibrahim ZM, Magdy Refaat Ahmed MR, Ahmed WAS. Prevalence and risk factors for female sexual dysfunction among Egyptian women. Arch Gynecol Obstet. 2013; 287: 1173-1180.
PMid:23274790 https : //doi.org/10.1007/s00404-012-267 7-8

[36] Goossens E, Norekval TM, Faerch J, et al. Moons, Sexual counseling of cardiac patients in Europe: culture matters, Int. J. Clin. Pract. 2011; 65(10): 1092-1099.

[37] Abd-Elsalam FG, Mahgoub NA, Ghoneim AH, et al. Chronic Obstructive Pulmonary Disease and sexual functioning among Women in Egypt. Egyptian Journal of Chest Diseases and Tuberculosis. 2015; 64: 551-556. https ://doi.org/10.1016/j.ejcdt. 2015 .02 .006

[38] Tehrani FR, Farahmand M, Simbar M, et al. Factors Associated with Sexual Dysfunction; A Population-Based Study in Iranian Reproductive-Age Women. Arch Iran Med. 2014; 17(10): 679-684.

[39] Esfehani JR, Fazel N, Dashti S, et al. Female Sexual Dysfunction and its Associated Risk Factors: An Epidemiological Study in the North-East of Iran. J Midwifery Reprod Health. 2016; 4(1): 498-505.

[40] Varghese KM, Bansal R, Kekre AN, et al. Sexual Dysfunction among Young Married Women in Southern India. International Urogynecology Journal. 2012; 23: 1771-1774. PMid:22531958 https://doi.org/10.1007/s00192-012-1782-3

[41] Wallwiener C, Seeger H, Mück A, et al. Prevalence of sexual dysfunction and the impact of contraception in female German medical students. The Journal of Sexual Medicine. 2010; 7(6): 2139-2148. PMid:20487241 https://doi .org/10.1111/j.1743-6109.20 $10.01742 . x$

[42] Burri A, Spector T. Recent and lifelong sexual dysfunction in a female UK population sample: prevalence and risk factors. The Journal of Sexual Medicine. 2011; 8(9): 2420-2430. PMid:21676186 https://doi.org/10.1111/j.1743-6109.2011.02341.x

[43] Umran O, Melike D. Effect of the Contraceptive Methods on Female Sexual Function. International Journal of Caring Sciences. 2016.

[44] Esposito K, Maiorino MI, Bellastella G, et al. Determinants of female sexual dysfunction in type 2 diabetes. Int J Impot Res. 2010; 22: 17984. PMid:20376056 https://doi .org/10.1038/ijir. 2010.6

[45] Khajehei M. Sexual function of women during the first year after childbirth: Effect of parity (giving birth after the 20th week of pregnancy), depression and relationship satisfaction. A published Thesis is presented for the Degree of Doctor of Philosophy of Curtin University. May 2013.

[46] Elyasi F, Kashi Z, Tasfieh B, et al. Sexual Dysfunction in Women with Type 2 Diabetes Mellitus. Iran J Med Sci. May 2015.

[47] AlMogbel TA, Amin HS, AlSaad SM, et al. Prevalence of sexual dysfunction in Saudi women with Type 2 diabetes: Is it affected by age, glycemic control or obesity?. Pak J Med Sci. 2017; 33(3): 732-737.

[48] Bakhta S, Najafib S. Body image and sexual dysfunctions: comparison between breast cancer patients and healthy women. Procedia Social and Behavioral Sciences. 2010.

[49] Reda MM, Ahmed HH, Moussa KHS, et al. Characteristics of an Egyptian sample of patients with female sexual dysfunction: a crosssectional study. Middle East Curr Psychiatry. 2013; 20: 242-250. https://doi.org/10.1097/01.XME.0000426293.86823.2f

[50] Amirkhani Z, Jangholi E, Ramezi P, et al. Prevalence Survey of Sexual Dysfunction among Women in the Reproductive Age Group Referred to the Islamic Azad University Hospital During 2011-2012. GMJ. 2014; 3(1): 14-19.

[51] El-Sakka AI, Sayed HM, Tayeb KA. Diabetes-associated androgen alteration in patients with erectile dysfunction. Int J Androl. 2008; 31: 602-608. PMid:17877718 https://doi.org/10.1111/j.13 65-2605.2007.00815.x

[52] Elnashar ARM, Ibrahim NH, Ahmed HEH, et al. Assessment of female sexual function in a group of uncircumcised obese Egyp- 
tian women. International Journal of Impotence Research. 2013; 27: 178-181. PMid:26155831 https://doi.org/10.1038/ijir .2015. 12

[53] El Atrash G, Ali MH, Abdelwahab HA, et al. The assessment of sexual dysfunction in Egyptian women with lower urinary tract symptoms. Arab Journal of Urology (Official Journal of the Arab Association of Urology). 2014.

[54] East C, Sherburn M, Nagle C, et al. Perineal pain following childbirth: Prevalence, effects on postnatal recovery and analgesia usage. Midwifery. 2012; 28(1): 93-97. PMid:21236531 https://doi.or g/10.1016/j.midw. 2010.11.009

[55] Rathfisch G, Dikencik B, Kizilkaya Beji N, et al. Effects of perineal trauma on postpartum sexual function. Journal of Advanced Nursing 2017; 66(12): 2640-2649. PMid:20735499 https ://doi .org/10 $.1111 / j .1365-2648.2010 .05428 . x$

[56] Pontiroli AE, Cortelazzi D, Morabito A. Female sexual dysfunction and diabetes: a systematic review and meta-analysis. J Sex Med 2013; 10: 1044-1051. PMid:23347454 https ://doi .org/10.111 $1 /$ jsm. 12065

[57] Both S, Ter Kuile M, Enzlin P, et al. Sexual response in women with type 1 diabetes mellitus: a controlled laboratory study measuring vaginal blood flow and subjective sexual arousal. Arch Sex Behav. 2015; 44: 1573-1587. PMid:26054485 https ://doi .org/10.100 7/s10508-015-0545-x

[58] Rosato E, Gigante A, Barbano B, et al. Clitoral blood flow in systemic sclerosis women: correlation with disease clinical variables and female sexual dysfunction. Rheumatology (Oxford). 2013; 52: 22382242. PMid:24030011 https ://doi.org/10.1093/rheumatolo gy/ket 305

[59] Afefy NA. Factors Associated with Female Sexual Problems among Women attending Cairo University Hospital. Journal of Biology, Agriculture, and Healthcare. 2015.

[60] Dettore D, Pucciarelli M, Santarnecchi E. Anxiety and female sexual functioning: an empirical study. J Sex Marital Ther. 2013; 39: 216-
240. PMid:23356511 https://doi.org/10.1080/0092623X.2 011.606879

[61] Kalmbach DA, Pillai V, Kingsberg SA, et al. The transaction between depression and anxiety symptoms and sexual functioning: a prospective study of premenopausal, healthy women. Arch Sex Behav. 2015; 44: 1635-1649. PMid:25403320 https://doi.org/10.1007/s1 0508-014-0381-4

[62] Zedan H, Hareadei AA, Abd-Elsayed AA, et al. Cigarette smoking, hypertension and diabetes mellitus as risk factors for erectile dysfunction in upper Egypt. EMHJ. 2010.

[63] Battaglia C, Battaglia B, Mancini F, et al. Cigarette smoking decreases the genital vascularization in young healthy, eumenorrheic women. J Sex Med. 2011; 8: 1717-1. PMid:21477023 https: //doi.org/10.1111/j.1743-6109.2011.02257.x

[64] Johannes CB, Clayton AH, Odom DM, et al. Distressing sexual problems in United States women revisited: prevalence after accounting for depression. J Clin Psychiatry. 2009; 70: 1698-1706. PMid:20141709 https://doi.org/10.4088/JCP.09m05390gr $\mathrm{y}$

[65] Grewal GS, Gill JS, Sidi H, et al. Prevalence and Risk Factors of Female Sexual Dysfunction among Healthcare Personnel in Malaysia. Comprehensive Psychiatry. 2014; 55: S17-S22.

[66] Bukhari Y, Mosli H, Batarfi A, et al. premature ejaculation: a study of prevalence, risk factors, and impact on sexual relationships among married men in Saudi Arabia. The 2nd Biennial Meeting of the Middle East Society for Sexual Medicine. 2013.

[67] Nahid Ghanbarzadeh N, Nadjafi-Semnani M, Reza Ghanbarzadeh M, et al. Female sexual dysfunction in Iran: a study of prevalence and risk factors. Arch Gynecol Obstet. 2013; 287: 533-539. PMid:23111907 https://doi.org/10.1007/s00404-012-2604-z

[68] Moel J, Buttner M, O'Hara M, et al. Sexual function in the postpartum period: effects of maternal depression and interpersonal psychotherapy treatment. Archives of Women's Mental Health. 2010; 13(6): 495-504. PMid:20549525 https://doi .org/10.1007/s0 0737-010-0168-0 